\title{
Born amplitudes in QCD from scalar diagrams*
}

\author{
Christian Schwinn ${ }^{\mathrm{a}}$ and Stefan Weinzierl ${ }^{\mathrm{b}}$ \\ anstitut für Theoretische Physik E, RWTH Aachen, D-52056 Aachen, Germany \\ ${ }^{\mathrm{b}}$ Institut für Physik, Johannes-Gutenberg-Universität, Staudingerweg 7, D-55099 Mainz, Germany
}

We review recent developments for the calculation of Born amplitudes in QCD. This includes the computation of gluon helicity amplitudes from MHV vertices and an approach based on scalar propagators and a set of threeand four-valent vertices. The latter easily generalizes to amplitudes with any number of quark pairs. The quarks may be massless or massive.

\section{Introduction}

At the CERN Large Hadron Collider (LHC) events with six or more partons in the final state will occur with significant rates and contribute to the background for electroweak physics and for searches for physics beyond the standard model. Already at tree level, the number of Feynman diagrams contributing to the relevant scattering amplitudes grows rapidly with the number of external legs, for instance over 34000 diagrams contribute to the eight gluon amplitude at Born level. More efficient methods to calculate multiparton scattering amplitudes have been developed, employing a decomposition of QCD Born amplitudes into gauge-independent color structures, the spinor helicity formalism and recursive techniques [1]. Using such methods it has been shown that helicity amplitudes for specific helicity combinations have a remarkably simple analytic form or vanish altogether $[2,3]$. In particular, a compact analytical form is known for the first non-vanishing amplitude - the so called maximalhelicity violating amplitude (MHV amplitude)where two gluons have the opposite helicity of the remaining ones.

Recently, new methods for further simplifications in the calculation of multiparticle amplitudes have been developed, initiated by insight gained from the transformation of the amplitudes to twistor space and a conjectured relation to a

\footnotetext{
*Talk given at QCD 05: 12th International QCD Conference, 4-9 Jul 2005, Montpellier, France
}

certain string model [4]. This development led to a construction by Cachazo, Svrček and Witten (CSW) [5] that expresses helicity amplitudes with arbitrary helicity configurations in terms of diagrams with vertices given by off-shell continuations of MHV-amplitudes, connected by scalar propagators. For certain helicity configurations, this formalism has led to much simpler expressions than the conventional expansion in terms of Feynman diagrams.

In this talk we will briefly review these recent developments and introduce a method [6] to compute Born amplitudes in QCD - including also massless or massive quarks - from scalar propagators and a set of three- and four-valent vertices.

In section 2 we recall the use of helicity methods and the color decomposition in the computation of born amplitudes. In section 3 we review the CSW construction for QCD amplitudes in terms of MHV vertices. In section 4 we introduce the formalism of scalar Feynman rules in QCD before discussing the extension to massive quarks in section 5 .

\section{Helicity methods in QCD}

An efficient algorithm for calculations in QCD starts with the separation of the color structure from the the kinematic and spinor structure of born amplitudes. This is accomplished using the so called color decomposition. In the pure gluonic case tree level amplitudes with $n$ external gluons may be written in the form 


$$
\begin{aligned}
& \mathcal{A}_{n}(1,2, \ldots, n) \\
& =g^{n-2} \sum_{\sigma \in S_{n} / Z_{n}} 2 \operatorname{Tr}\left(T^{a_{\sigma(1)}} \ldots T^{a_{\sigma(n)}}\right) \\
& \\
& A_{n}(\sigma(1), \ldots, \sigma(n)),
\end{aligned}
$$

where the sum is over all non-cyclic permutations of the external gluon legs. The color structure is contained in group theoretical factors given by traces over the generators $T^{a}$ of the fundamental representation of $S U(3)$. The quantities $A_{n}(\sigma(1), \ldots, \sigma(n))$, called the partial amplitudes, contain the kinematic information and are separately gauge invariant. They are color-ordered, e.g. only diagrams with a particular cyclic ordering of the gluons contribute. For amplitudes containing external quarks, similar decompositions exist.

The calculation of the partial amplitudes is simplified by the spinor helicity formalism [7]. The basic ingredients are two-component Weyl spinors $|p+\rangle$ and $|p-\rangle$ that are defined as solutions of the Weyl equations

$$
\sigma^{\mu} p_{\mu}|p-\rangle=0 \quad, \quad \bar{\sigma}^{\mu} p_{\mu}|p+\rangle=0
$$

where $\sigma^{\mu}=(1,-\vec{\sigma})$ and $\bar{\sigma}^{\mu}=(1, \vec{\sigma})$. Antisymmetric spinor products can be defined by

$$
\langle p k\rangle=\langle p-\mid k+\rangle \quad, \quad[p k]=\langle p+\mid k-\rangle
$$

Gluons can be included in the spinor formalism by expressing the polarization vectors of the external gluons in terms of Weyl-Spinors:

$$
\epsilon_{\mu}^{ \pm}(k, q)= \pm \frac{\left\langle q \mp\left|\gamma_{\mu}\right| k \mp\right\rangle}{\sqrt{2}\langle q \mp \mid k \pm\rangle}
$$

where $q$ is an arbitrary light-like reference momentum. On can show that different choices of $q$ correspond to different gauge choices for the external gluons.

The helicity formalism allowed to derive remarkably simple analytic formula for helicity amplitudes with specific helicity combinations $[2,3]$. In particular, the pure gluon amplitude vanishes if all gluons have the same helicity, or if all gluons except one have the same helicity. The first non-vanishing gluon amplitude is obtained if $n-2$ gluon have one type of helicity, and 2 gluons the other type. This so called MHV amplitude is given by the Parke-Taylor formula [2]

$$
\begin{aligned}
& A_{n}\left(1^{+}, \ldots, i^{-}, \ldots, j^{-}, \ldots n^{+}\right) \\
& =\mathrm{i} 2^{n / 2-1} \frac{\langle i j\rangle^{4}}{\langle 12\rangle\langle 23\rangle \ldots\langle(n-1) n\rangle\langle n 1\rangle}
\end{aligned}
$$

This was proven later by Berends and Giele [3] using recursive techniques. For reviews of further developments and applications of the helicity formalism see e.g. [1].

\section{QCD amplitudes from MHV diagrams}

The fact, that the MHV amplitudes for an arbitrary number of external gluons take the simple form (5) motivated a number of authors to search for an explanation outside the usual Feynman diagrammatic methods [8]. Recently Witten related scattering amplitudes of (super-) Yang-Mills theory to a certain string model in Twistor space [4]. Motivated by this connection, on the field theoretic side Cachazo, Svrček and Witten [5] subsequently proposed a construction of all tree-level QCD amplitudes in terms of MHV vertices (5) as building blocks. This construction allows a considerable reduction of the computational effort for multiparton amplitudes compared to the usual expansion in terms of Feynman diagrams.

As a first step, an appropriate off-shell continuation of the MHV amplitudes has to be introduced. Since a spacelike or timelike four momentum cannot be decomposed into just two Weyl spinors, the formula (5) cannot be used for offshell momenta in a straightforward way. Here we chose the prescription of [9] that is slightly different from the original CSW prescription. To translate an off-shell momentum into spinor language, one decomposes an arbitrary off-shell momentum into two lightlike vectors according to

$$
k^{\mu}=k^{b \mu}+\frac{k^{2}}{2(k \cdot q)} q^{\mu}
$$

where $q^{\mu}$ is a fixed, lightlike reference four-vector. We can now associate two-component spinors $\left|k^{b}+\right\rangle$ and $\left|k^{b}-\right\rangle$ with the projected momentum $k^{b \mu}$. An MHV vertex including an off-shell gluon with momentum $k_{l}$ can now be defined using the 
spinor corresponding to the projected momentum $k_{l}^{b}$, i.e. by replacing $\langle l n\rangle \rightarrow\left\langle l^{b} n\right\rangle$ in the expression (5). If all external gluons are off-shell, the MHV vertex is hence given by

$$
\begin{aligned}
& V_{\mathrm{CSW}}\left(1^{+}, \ldots, i^{-}, \ldots, j^{-} \ldots, n^{+}\right) \\
& \quad=A\left(1^{b+}, \ldots, i^{b-}, \ldots, j^{b-} \ldots, n^{b+}\right)
\end{aligned}
$$

The CSW prescription then expresses an arbitrary QCD amplitude with $n^{-}$external negative helicity gluons in terms of $d=n^{-}-1$ MHV vertices, where scalar propagators connect + and labels.

As an example, consider the so called nextto maximal helicity violating (NMHV) amplitudes with three gluons of negative helicity. For simplicity, we consider the amplitudes $A\left(1^{-}, 2^{-}, 3^{+}, \ldots n^{-}\right)$where the three negative helicity gluons are adjacent. The algorithm begins by distributing the three external negative helicity gluons in all possible ways over two MHV vertices. For our example, there are three partitions $((1,2), n),((1,(2, n))$ and $(2,(n, 1))$. Next, connect the vertices by a scalar propagator and distribute the positive helicity gluons in all possible ways compatible with the cyclic color ordering. In the present example, the combination $((1,(2, n))$ gives a vanishing contribution so only two topologies contribute. The amplitude is given by a sum over the possible insertions of the positive helicity gluons:

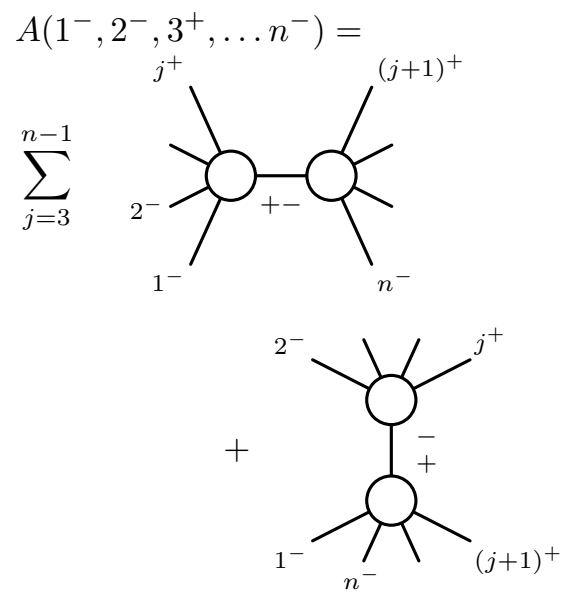

In this way, the $n$ gluon NMHV amplitude is expressed in terms of $2(n-3)$ CSW diagrams, re- ducing the growth of computational effort with the number of external particles drastically compared to the usual expansion in terms of Feynman diagrams. A closed expression for all NMHV amplitudes for gluons - including an arbitrary placement of the three negative helicity gluons - has been found using the CSW formalism in [9].

Based on so called on-shell recursion relations [10] both indirect arguments [11] for the validity of the CSW rules and a direct derivation [12] have been given. The relation to ordinary Feynman diagrams, however, remains to be clarified. For further applications of the CSW formalism and extensions to fermions see e.g. [13]. The application to loop diagrams has been initiated in [14]. For a review and references to subsequent developments and the connection to twistor string theory see e.g. [15].

\section{Scalar Feynman rules for QCD}

So far, the CSW construction described in the previous section is applicable only for massless quarks. Also, an understanding in terms of conventional Feynman diagrams would be desirable. As a first step towards these issues, in [6] we have introduced scalar diagrammatic rules for QCD partial amplitudes, that use a similar language as the CSW construction. As all propagators are scalars no contraction of Lorentz- or spinorindices is present any more. This makes our method also well suited for a fast implementation on a computer.

We employ the decomposition of an off-shell momentum into two lightlike vectors according to $(6)$. In the following, we use spinor products defined by:

$$
\langle a b\rangle=\left\langle a^{b}-\mid b^{b}+\right\rangle,[a b]=\left\langle a^{b}+\mid b^{b}-\right\rangle
$$

We define the off-shell continuation of polarization vectors for gluons using the Weyl-spinors $\left|k^{b}\right\rangle$ associated with the projection $k^{b \mu}$ of the off-shell gluon momentum:

$$
\epsilon_{\mu}^{ \pm}(q)= \pm \frac{\left\langle q \mp\left|\gamma_{\mu}\right| k^{b} \mp\right\rangle}{\sqrt{2}\left\langle q \mp \mid k^{b} \pm\right\rangle}
$$

These definitions allow to rewrite the gluon propagator in the light-cone gauge as a sum over the 
polarization vector (10) and an additional instantaneous term:

$$
\begin{aligned}
& \frac{\mathrm{i}}{k^{2}}\left(-g_{\mu \nu}+\frac{k_{\mu} q_{\nu}+k_{\nu} q_{\mu}}{q \cdot k}\right) \\
& =\frac{\mathrm{i}}{k^{2}} \sum_{\sigma= \pm} \epsilon_{\mu}^{\sigma}\left(k^{b}, q\right) \epsilon_{\mu}^{* \sigma}\left(k^{b}, q\right)+\frac{\mathrm{i}}{(q \cdot k)^{2}} q_{\mu} q_{\nu}
\end{aligned}
$$

As noted earlier $[16,9]$, the instantaneous term can be absorbed into a redefinition of the fourgluon vertex, without generating vertices with more than four gluons. Similar results can also be obtained by eliminating the unphysical degrees of freedom in the Lagrangian using the equations of motion [17].

Contracting the polarization vectors with the vertices, one obtains diagrammatic rules involving only scalar propagators $\mathrm{i} / k^{2}$ connecting opposite helicities and vertices obtained by contracting the standard Feynman rules with the polarization vectors. We call these vertices "primitive vertices" to distinguish them from the vertices of the standard Feynman rules on the one hand, and from the MHV-vertices on the other hand. The nonvanishing three gluon vertices obtained in this way are given by [6]

$$
\begin{aligned}
& V_{3}\left(1^{-}, 2^{-}, 3^{+}\right)=\mathrm{i} \sqrt{2} \frac{\langle 12\rangle^{4}}{\langle 12\rangle\langle 23\rangle\langle 31\rangle} \\
& V_{3}\left(1^{+}, 2^{+}, 3^{-}\right)=\quad \mathrm{i} \sqrt{2} \frac{[21]^{4}}{[32][21][13]}
\end{aligned}
$$

and cyclic permutations thereof. The modified four gluon vertices can be found in [6].

To gain insight in the structure of scalar diagrams obtained using the rules defined in this section, one can define the degree of a vertex or of an amplitude as the number of "-"-labels minus one. In the diagrammatic rules, only primitive vertices of degree zero and one occur. Furthermore, the degree of an amplitude is exactly the sum of the degrees of the primitive vertices [6]. Therefore, one can see the structure of the CSW prescription emerge from our diagrammatic approach: a MHV amplitude is of degree one and contains exactly one primitive vertex of degree one, which is dressed up in all possible ways with degree zero vertices. Similar, a pure gluon amplitude with three gluons of negative helicities is of degree two and contains two vertices of degree one, which again are combined in all possible ways with degree zero vertices.

\section{Scalar rules for massive quarks}

Several methods exist to incorporate massive fermions into the helicity formalism $[18,19]$. Here we will introduce an off-shell continuation, using the same projection (6) as for the gluon polarization vectors:

$$
\begin{aligned}
& u(+)=\frac{\not p+m}{\left\langle p^{b} q\right\rangle}|q+\rangle=\left|p^{b}-\right\rangle+\frac{m}{\left\langle p^{b} q\right\rangle}|q+\rangle \\
& u(-)=\frac{\not p+m}{\left[p^{b} q\right]}|q-\rangle=\left|p^{b}+\right\rangle+\frac{m}{\left[p^{b} q\right]}|q-\rangle
\end{aligned}
$$

The normalization is chosen in order to allow for a smooth massless limit. The spinors (13) are eigenstates of $\gamma^{5} \$$ with the spin vector [18]

$$
s^{\mu}=\frac{p^{\mu}}{m}-\frac{m}{(p \cdot q)} q^{\mu}
$$

Therfore the reference momentum is not an unphysical quantity that has to drop out in the final result for the helicity amplitude, as in the case of the gluon polarization vectors, but rather defines the quantization axis of the quark spin.

Similarly to the case of the gluon propagator, the definitions (13) allow to rewrite the quark propagator as a sum over off-shell spinors and an additional instantaneous term:

$\frac{\mathrm{i}(\not p+m)}{p^{2}-m^{2}}=\frac{\mathrm{i}}{p^{2}-m^{2}} \sum_{\lambda} u(-\lambda) \bar{u}(\lambda)+\mathrm{i} \frac{\not q}{2(p \cdot q)}$

Again the instantaneous contribution can be absorbed by introducing only quartic vertices [6], leading to additional vertices involving two quarks and two gluons or four quarks. In this way, also massive quarks can be described in terms of scalar propagators i/ $\left(k^{2}-m^{2}\right)$ connecting opposite helicities. The primitive vertices obtained by contracting the spinors into the ordinary Feynman rules include vertices present both for mass- 
less and massive quarks like

$$
\begin{aligned}
& V_{3}\left(1_{q}^{+}, 2_{\bar{q}}^{-}, 3^{+}\right)=\mathrm{i} \sqrt{2} \frac{[13]^{2}}{[12]} \\
& V_{3}\left(1_{q}^{-}, 2_{\bar{q}}^{+}, 3^{-}\right)=\mathrm{i} \sqrt{2} \frac{\langle 31\rangle^{2}}{\langle 21\rangle}
\end{aligned}
$$

In addition, there are primitive vertices involving a helicity flip along the quark line that vanish for massless quarks:

$$
\begin{aligned}
& V_{3}\left(1_{q}^{+}, 2_{\bar{q}}^{+}, 3^{-}\right)=\mathrm{i} \sqrt{2} m \frac{[12]^{2}}{[23][31]} \\
& V_{3}\left(1_{q}^{-}, 2_{\bar{q}}^{-}, 3^{+}\right)=-\mathrm{i} \sqrt{2} m \frac{\langle 12\rangle^{2}}{\langle 23\rangle\langle 31\rangle}
\end{aligned}
$$

The four-valent vertices can be found in [6].

In the amplitudes for massive quarks there are two new features compared to the massless case. First there are helicity configurations, which vanish in the simultaneous massless and on-shell limit, but remain non-zero in the on-shell limit for non-zero masses. Examples of this kind are amplitudes with only positive helicity gluons:

$$
A_{n}\left(1_{q}^{+}, 2_{\bar{q}}^{-}, 3^{+}, \ldots, n^{+}\right) .
$$

Secondly, there are the helicity flip vertices (17) which are proportional to the mass and vanish therefore in the massless limit. We note that $V_{3}\left(1_{q}^{+}, 2_{\bar{q}}^{+}, 3^{-}\right)$is a degree zero vertex, whereas $V_{3}\left(1_{q}^{-}, 2_{\bar{q}}^{-}, 3^{+}\right)$has degree one. This allows to determine the maximal number of flip-vertices in an amplitude with a given degree. Let us consider an amplitude with one massive quark pair. We first consider the helicity configuration $q^{+} \bar{q}^{-}$. Along the massive quark line we must have an equal number of helicity flips induced by $V_{3}\left(1_{q}^{+}, 2_{\bar{q}}^{+}, 3^{-}\right)$ and $V_{3}\left(1_{\bar{q}}^{-}, 2_{\bar{q}}^{-}, 3^{+}\right)$. Since the latter vertex is of degree one, the total number of helicity flips $f$ is bounded by the degree $d$ of the amplitude:

$$
f \leq 2 d
$$

As an example it follows immediately from this bound that amplitudes of the form (18) cannot contain a helicity flip. A similar argument applies to the helicity configuration $q^{+} \bar{q}^{+}$. Here we have along the fermion line at least one helicity flip of degree zero together with at most $d$ additional pairs of degree one and zero helicity flips. So the total number is bounded by

$$
f \leq 2 d+1 .
$$

For the helicity configuration $q^{-} \bar{q}^{-}$there is at least one flip of degree one, leaving at most $2(d-$ 1) additional helicity flips so the total number is bounded by

$$
f \leq 2 d-1 .
$$

While a closed expression for amplitudes with a massive quark pair and an arbitrary number of positive helicity gluons has been found recently using a different choice of reference momenta for the massive quarks [19], we expect the formalism described in this section and the classification of the amplitudes given above to be useful for the calculation of amplitudes with massive quarks with other helicity configurations.

\section{Conclusions}

After a review of spinor helicity methods in QCD we have sketched the CSW construction for expressing Born amplitudes in terms of MHV vertices, connected by scalar propagators.

We have then reviewed a method to calculate born amplitudes from a set of scalar, complex valued three- and four-valent vertices. Our approach is not restricted to gluons only, but treats gluons, massless quarks and massive quarks on almost equal footing. It is possible to assign to each vertex a degree, given by the number of negative helicities minus one. Only vertices of degree zero and one occur and the number of degree one vertices in a diagram is equal to the number of MHV vertices in the CSW construction. In the case of massive quarks, it is possible to bound the number of helicity flips in terms of the degree of the amplitude.

We hope that our method will be useful to obtain closed expressions for helicity amplitudes involving massive quarks and to get further insight into the CSW construction. Work in this direction is in progress. Our method is also well suited for a fast implementation on a computer. 


\section{Acknowledgments}

The work of CS has been supported by the Deutsche Forschungsgemeinschaft through the Graduiertenkolleg 'Eichtheorien' at Mainz University.

\section{REFERENCES}

1. M.L. Mangano and S.J. Parke, Phys. Rept. 200 (1991) 301; L.J. Dixon, QCD and beyond: Proceedings of TASI 95, edited by D. Soper, pp. 539-584, 1996, hep-ph/9601359.

2. S.J. Parke and T.R. Taylor, Phys. Rev. Lett. 56 (1986) 2459.

3. F.A. Berends and W.T. Giele, Nucl. Phys. B306 (1988) 759.

4. E. Witten, Commun. Math. Phys. 252 (2004) 189, hep-th/0312171.

5. F. Cachazo, P. Svrcek and E. Witten, JHEP 09 (2004) 006, hep-th/0403047.

6. C. Schwinn and S. Weinzierl, JHEP 05 (2005) 006, hep-th/0503015.

7. F.A. Berends et al., Phys. Lett. B103 (1981) 124; P. De Causmaecker et al., Nucl. Phys. B206 (1982) 53; J.F. Gunion and Z. Kunszt, Phys. Lett. B161 (1985) 333; Z. Xu, D.H. Zhang and L. Chang, Nucl. Phys. B291 (1987) 392.

8. V.P. Nair, Phys. Lett. B214 (1988) 215; W.A. Bardeen, Prog. Theor. Phys. Suppl. 123 (1996) 1; D. Cangemi, Nucl. Phys. B484 (1997) 521, hep-th/9605208; G. Chalmers and W. Siegel, Phys. Rev. D54 (1996) 7628, hep-th/9606061.

9. D.A. Kosower, Phys. Rev. D71 (2005) 045007, hep-th/0406175.

10. R. Britto, F. Cachazo and B. Feng, Nucl. Phys. B715 (2005) 499, hep-th/0412308.

11. R. Britto et al., Phys. Rev. Lett. 94 (2005) 181602, hep-th/0501052.

12. K. Risager, (2005), hep-th/0508206.

13. G. Georgiou and V.V. Khoze, JHEP 05 (2004) 070, hep-th/0404072; G. Georgiou, E.W.N. Glover and V.V. Khoze, JHEP 07 (2004) 048, hep-th/0407027; J.B. Wu and C.J. Zhu, JHEP 07 (2004) 032, hepth/0406085; J.B. Wu and C.J. Zhu, JHEP 09 (2004) 063, hep-th/0406146; M.x. Luo and C.k. Wen, JHEP 11 (2004) 004, hepth/0410045; I. Bena, Z. Bern and D.A. Kosower, Phys. Rev. D71 (2005) 045008, hepth/0406133.

14. F. Cachazo, P. Svrcek and E. Witten, JHEP 10 (2004) 074, hep-th/0406177; F. Cachazo, P. Svrcek and E. Witten, JHEP 10 (2004) 077, hep-th/0409245; A. Brandhuber, B. Spence and G. Travaglini, Nucl. Phys. B706 (2005) 150, hep-th/0407214; I. Bena et al., Phys. Rev. D71 (2005) 106010, hepth/0410054.

15. F. Cachazo and P. Svrcek, (2005), hepth/0504194.

16. D.A. Kosower, Nucl. Phys. B335 (1990) 23.

17. G. Chalmers and W. Siegel, Phys. Rev. D59 (1999) 045013, hep-ph/9801220; W. Siegel, Fields, 1999, hep-th/9912205.

18. R. Kleiss and W.J. Stirling, Nucl. Phys. B262 (1985) 235; A. Ballestrero and E. Maina, Phys. Lett. B350 (1995) 225, hepph/9403244; S. Dittmaier, Phys. Rev. D59 (1999) 016007, hep-ph/9805445; J. van der Heide et al., Phys. Rev. D62 (2000) 074025, hep-ph/0003318.

19. G. Rodrigo, (2005), hep-ph/0508138. 